\title{
GLIS3 is indispensable for TSH/TSHR-dependent thyroid hormone biosynthesis and follicular cell proliferation
}

\author{
Hong Soon Kang, ${ }^{1}$ Dhirendra Kumar, ${ }^{2}$ Grace Liao, ${ }^{1}$ Kristin Lichti-Kaiser, ${ }^{1}$ Kevin Gerrish, ${ }^{3}$ Xiao-Hui Liao, ${ }^{4}$ Samuel Refetoff, ${ }^{4,5}$ \\ Raja Jothi, ${ }^{2}$ and Anton M. Jetten ${ }^{1}$ \\ IImmunity, Inflammation and Disease Laboratory, ${ }^{2}$ Epigenetics and Stem Cell Biology Laboratory, and ${ }^{3}$ Molecular Cenomics Core, National Institute of Environmental Health Sciences (NIEHS), NIH, \\ Research Triangle Park, North Carolina, USA. ${ }^{4}$ Department of Medicine, and ${ }^{5}$ Department of Pediatrics and Committee on Cenetics, The University of Chicago, Chicago, Illinois, USA.
}

\begin{abstract}
Deficiency in Krüppel-like zinc finger transcription factor GLI-similar 3 (GLIS3) in humans is associated with the development of congenital hypothyroidism. However, the functions of CLIS3 in the thyroid gland and the mechanism by which GLIS3 dysfunction causes hypothyroidism are unknown. In the current study, we demonstrate that GLIS3 acts downstream of thyroid-stimulating hormone (TSH) and TSH receptor (TSHR) and is indispensable for TSH/TSHR-mediated proliferation of thyroid follicular cells and biosynthesis of thyroid hormone. Using ChIP-Seq and promoter analysis, we demonstrate that CLIS3 is critical for the transcriptional activation of several genes required for thyroid hormone biosynthesis, including the iodide transporters Nis and Pds, both of which showed enhanced GLIS3 binding at their promoters. The repression of cell proliferation of GLIS3-deficient thyroid follicular cells was due to the inhibition of TSH-mediated activation of the mTOR complex 1/ribosomal protein S6 (mTORC1/RPS6) pathway as well as the reduced expression of several cell division-related genes regulated directly by GLIS3. Consequently, CLIS3 deficiency in a murine model prevented the development of goiter as well as the induction of inflammatory and fibrotic genes during chronic elevation of circulating TSH. Our study identifies CLIS3 as a key regulator of TSH/TSHR-mediated thyroid hormone biosynthesis and proliferation of thyroid follicular cells and uncovers a mechanism by which CLIS3 deficiency causes neonatal hypothyroidism and prevents goiter development.
\end{abstract}

\section{Introduction}

Thyroid hormones (THs), thyroxine (T4), and triiodothyronine (T3) play a critical role in the regulation of many developmental and metabolic processes. TH biosynthesis and secretion is controlled by the hypothalamus-pituitary-thyroid axis and is maintained constant via negative feedback regulation $(1,2)$. Thyroidstimulating hormone (TSH), produced by thyrotrophic cells in the pituitary gland, stimulates $\mathrm{TH}$ biosynthesis and secretion, while the production of TSH is regulated by the hypothalamic TSHreleasing hormone and modulated by $\mathrm{TH}$ via a negative feedback loop. Interaction of TSH with the TSH receptor (TSHR), a G proteincoupled receptor $\left(\mathrm{G} \alpha_{\mathrm{q}} / \mathrm{G} \alpha_{11}\right)$, leads to activation of several kinase signaling cascades, including PKA, PKC, AMPK, and the PI3K/ mTOR complex 1 (mTORC1) pathways (2-8). Through these pathways, TSH regulates the expression of genes that are associated with thyroid gland development and $\mathrm{TH}$ biosynthesis and secretion, including thyroid peroxidase (TPO), and the $\mathrm{Na}^{+} / \mathrm{I}^{-}$symporter (NIS; SLC5A5) and iodide transporter pendrin (PDS, also referred to as $S L C 26 A 4)(7,9,10)$. Prolonged TSH stimulation causes proliferation of thyroid follicular cells, leading to enlargement of the thyroid gland (goiter) $(6,8,11)$.

Congenital hypothyroidism is the most common inborn endocrine disorder, with a prevalence of 1 in 3,000 to 4,000 newborn

Conflict of interest: The authors have declared that no conflict of interest exists. Submitted: April 4, 2017; Accepted: September 12, 2017.

Reference information: / Clin Invest. 2017;127(12):4326-4337.

https://doi.org/10.1172/JCI94417. infants, that is most commonly due to abnormalities in thyroid development (thyroid dysgenesis) and includes mutations in thyroid transcription factor 1 (TTF1, also referred to as NKX2.1), TTF2 (also referred to as FOXE1), and paired box 8 (PAX8), genes that are critical for thyroid development (12-18). About $15 \%$ of the cases with congenital hypothyroidism are related to defects in $\mathrm{TH}$ biosynthesis (dyshormonogenesis) and include genetic alterations in genes critical for TH synthesis, such as TPO, TG, NIS, dual oxidase 2 and $A 2$ (DUOX2 and DUOXA2), PDS, iodotyrosine deiodinase (IYD), and TSHR (17, 19-23).

Recent studies have linked mutations in the Krüppel-like zinc finger transcription factor GLI-similar 3 (GLIS3) gene to a syndrome characterized by neonatal diabetes and congenital hypothyroidism that can include a wider spectrum of abnormalities, such as mild mental retardation and polycystic kidney disease (24-29). Similar phenotypes have been observed in Glis3-deficient mice (29-35). Recently, an association was found between GLIS3 variants and congenital hypothyroidism (36). In patients with mutations in GLIS3, the congenital thyroid phenotype has been variable and shown to range from aplasia/dysplasia to dyshormonogenesis (24-27, 36, 37). However, GLIS3's function in the thyroid gland and the mechanisms by which GLIS3 deficiency leads to the development of congenital hypothyroidism have remained elusive. In this report, we study the molecular and physiological functions of GLIS3 in the thyroid gland and uncover the mechanism by which GLIS3 deficiency leads to congenital hypothyroidism and prevents the development of goiter. We show that GLIS3 acts downstream of TSH/TSHR and is essential for the induction of TSH/ 
A

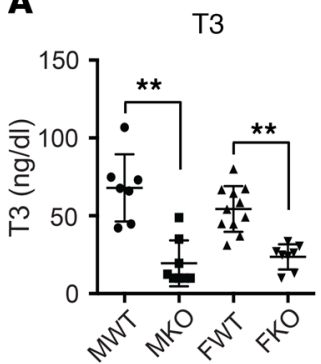

T4



TSH

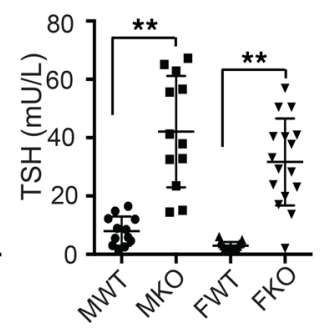

D



B

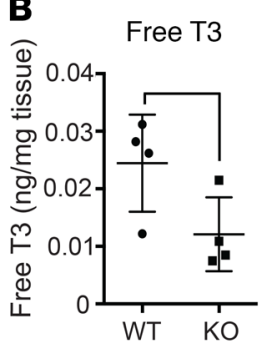

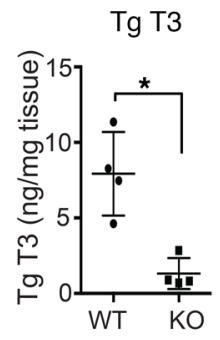
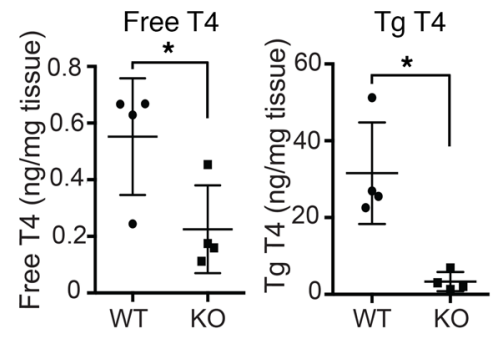

E



Figure 1. TH levels are reduced and TSH levels elevated in Glis3KO mice. (A) Comparison of serum T3, T4, and TSH levels between 1-month-old male and female WT (MWT and FWT, respectively) and Clis3KO (MKO and FKO, respectively) mice $(n=7-16)$. (B) The content of free T3, free T4, and thyroglobulinbound T3 and T4 (Tg T3 and Tg T4, respectively) were analyzed in thyroid tissues from 1-month-old male and female WT and Glis3KO mice $(n=4)$. The content of hormones was normalized to tissue weight. Data are shown as mean \pm SEM. (C) Glis3 is highly expressed in the thyroid. Levels of Clis3 mRNA in the liver, thyroid gland, pancreatic islets, and P7 testis were analyzed by QRT-PCR. Levels of expression were examined in tissues from at least 2 different mice. (D) Sections of thyroids from 1-week-old Glis3-ECFP mice were examined by immunohistochemistry with anti-GFP and anti-PECAM1 antibodies as described in Methods. Scale bar: $50 \mu \mathrm{m}$. (E) Thyroid tissues from 1-, 2-, and 4-week-old Glis3-EGFP mice were examined with antibodies against GFP and PAX8. Scale bar: $50 \mu \mathrm{m}$. ${ }^{*} P<0.05 ;{ }^{*} P<0.001$, Student's $t$ test..

TSHR-mediated TH biosynthesis and the proliferation of thyroid follicular cells. Based on the regulation of these critical functions by GLIS3, the GLIS3 pathway may provide a potential therapeutic target in the management of several thyroid pathologies.

\section{Results}

Development of hypothyroidism in Glis3-deficient mice. To gain insights into the function of GLIS3 in the regulation of TH homeostasis and the development of hypothyroidism, the serum levels of T3, T4, and TSH in WT and Glis3-deficient (Glis3KO) mice were measured. The serum levels of both $\mathrm{T} 3$ and $\mathrm{T} 4$ were significantly lower in male and female Glis3KO mice compared with those of WT mice; in contrast, serum levels of TSH were greatly elevated in Glis3KO mice (Figure 1A). Moreover, levels of both free and thyroglobulin-bound T3 and T4 were dramatically decreased in the thyroid glands of Glis3KO mice compared with those of WT mice (Figure 1B), suggesting a major role for GLIS3 in the regulation of TH biosynthesis. TH plays a critical role in growth (20, 38, 39); therefore, the reduction in TH levels might, at least in part, be responsible for the observed growth retardation in 3-weekold Glis3KO mice (Supplemental Figure 1; supplemental mate- rial available online with this article; https://doi.org/10.1172/ JCI94417DS1).

To investigate whether the regulation of $\mathrm{TH}$ biosynthesis by GLIS3 is intrinsic to the control of thyroid gland function, we examined the expression of GLIS3 in the thyroid gland. The level of Glis 3 expression in the thyroid gland was almost equivalent to that in pancreatic islets and neonatal testis (Figure 1C), tissues in which Glis3 is highly expressed $(31,33)$. Moreover, analysis of the (sub)cellular localization of GLIS3 protein in the thyroid of Glis3-EGFP mice showed that GLIS3-EGFP is not expressed in $\mathrm{PECAM}^{+}$endothelial cells (Figure 1D), but is expressed only in the nucleus of cells staining positively for the thyroid follicular cell marker PAX8 (Figure 1E). These data suggest a direct relationship between the decrease in $\mathrm{TH}$ production in thyroid glands of Glis3KO mice and loss of GLIS3 in thyroid follicular cells.

GLIS3 is a key regulator of thyroid follicular cell proliferation. To investigate whether loss of GLIS3 function affected thyroid gland development and morphology, histological sections of thyroid gland from WT and Glis3KO mice were analyzed. Our data showed that, although the general architecture of the thyroid glands was very similar, the average size of the follicles was sig- 
A

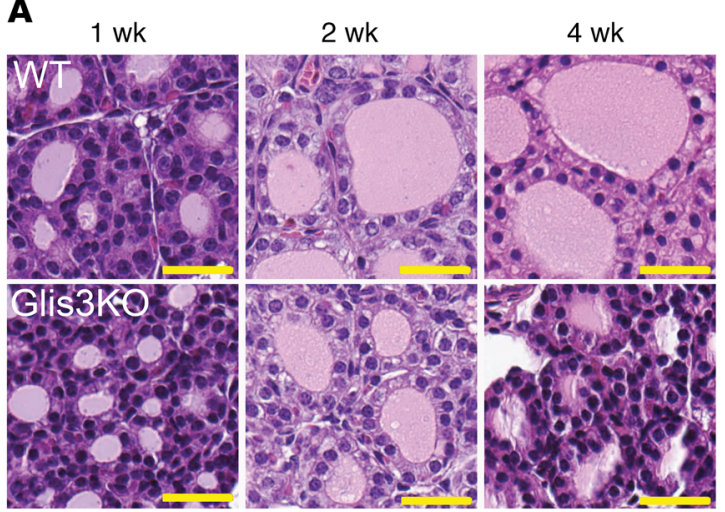

B



C

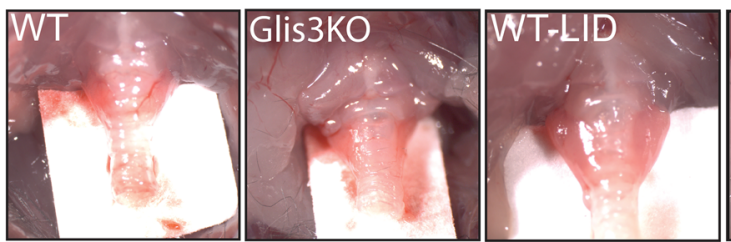

D
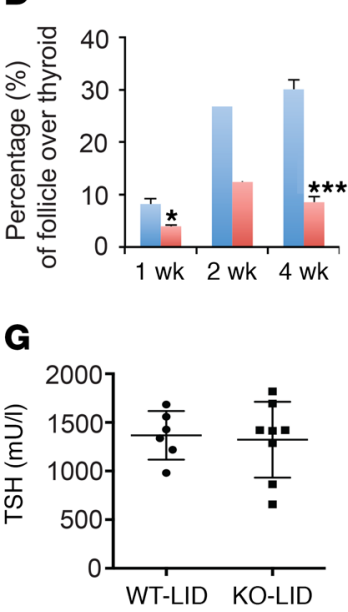

E

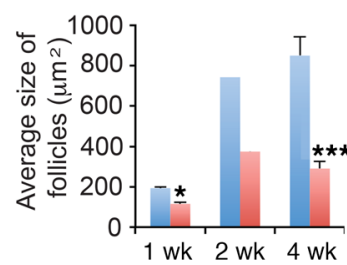

H





$\mathbf{F}$

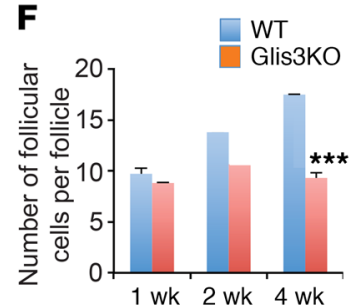

I

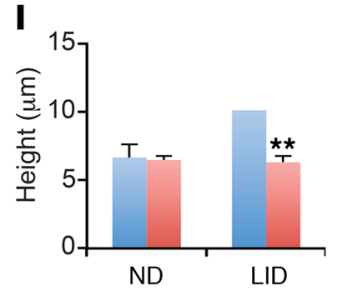

Figure 2. GLIS3 deficiency inhibits thyroid follicular cell proliferation and prevents goiter development in mice fed a LID. (A) Thyroid glands from 1-, 2-, and 4-week-old WT and Glis3KO mice and (B) WT-LID and KO-LID mice were examined by H\&E staining. Scale bars: $30 \mu \mathrm{m}$. (C) Representative thyroid glands with trachea from 4-week-old WT and Clis3KO mice and WT-LID and KO-LID mice. (D-F) Ratios of the surface area of thyroid follicles over total surface area of the thyroid (D), the average size of the follicles $(\mathbf{E})$, and the average number of follicular cells per follicle (F) were analyzed in thyroid glands from 1-, 2-, and 4-week-old WT and Glis3KO mice. $n \geq 2$ for each group. (G and $\mathbf{H}$ ) WT and Glis3KO mice were fed a LID for 6 days before serum TSH levels (C) and the relative weight of the thyroids (H) were determined. (I) Thyroid follicular cells from KO-LID mice did not become hypertrophic. Comparison of the average thyroid follicular cell size between WT and Glis3KO mice. $n \geq 2$ for each group. Data are shown as mean \pm SEM. ${ }^{*} P<0.05 ;{ }^{* *} P<0.001 ;{ }^{* * *} P<0.0001$, Student's $t$ test.

nificantly smaller in 1-week-old Glis3KO mice compared with those in WT mice (Figure 2A). Changes in thyroid architecture and follicle size became more apparent at 4 weeks (Figure 2, A and D). The thyroid glands from Glis3KO mice were smaller and the number of follicular cells/follicle was significantly lower compared with WT mice (Figure 2, A, C-F). No difference in apoptotic (Supplemental Figure 2) or autophagic (not shown) cell death was observed between WT and Glis3KO thyroid glands. Consistent with histological analysis, serum TH levels were not significantly different from those of WT and Glis3KO mice at 1 week old (Supplemental Figure 3). These observations suggest that GLIS3 does not play a major role during prenatal thyroid gland development, but instead controls the size of the follicles postnatally by regulating the proliferation of thyroid follicular cells.

Low-iodine diet (LID) is known to greatly increase the serum levels of TSH, which through its activation of TSHR, leads to increased proliferation of thyroid follicular cells and enlargement of the thyroid gland $(4,7)$. Consistent with previous studies, we found that WT mice fed a LID (WT-LID) had elevated levels of circulating TSH (compare Figure 1A and Figure 2G) and developed goiter. The relative thyroid weight increased 2-fold, and the thyroid follicular cells became hypertrophic (Figure 2, C, H, and I), which is typically associated with goiter (40). TSH levels, which were already greatly elevated in Glis3KO mice fed a normal diet (ND) (Figure $1 \mathrm{~A})$, became even higher in Glis3KO mice fed a LID (KO-LID), reaching levels comparable to those in WT-LID mice (Figure 2G). However, in contrast to WT-LID mice, KO-LID mice did not develop an enlarged thyroid; the relative weight of the KO-LID thyroid gland was not significantly increased, and follicular cells did not become hypertrophic (Figure 2, B, C, H, and I). These results suggest that GLIS3 is a critical regulator of the proliferation of thyroid follicular cells downstream of TSH/TSHR. This conclusion was supported by 5-ethynyl-2'-deoxyuridine (EdU) incorporation analyses showing that the percentage of $\mathrm{EdU}^{+} \mathrm{PAX}^{+}$thyroid follicular cells was greatly reduced in the thyroid glands from KO-LID mice (Figure 3, A and B).

Regulation of cell proliferation genes by GLIS3. To obtain further insights into the mechanisms by which GLIS3 deficiency leads to reduced follicular cell proliferation and TH biosynthesis, we compared the gene expression profiles of thyroid glands from 4-week-old WT and Glis3KO mice fed either a ND or a LID by microarray and RNA-Seq analysis. Gene ontology (GO) and pathway analysis (http://www.genome.jp/ $\mathrm{kegg} /$ ) showed that genes downregulated in thyroid glands from Glis3KO mice fed a ND were enriched for cell cycle and extracellular matrix-related (ECMrelated) genes (Figure $3 \mathrm{C}$ and Supplemental Table $1, \mathrm{~A}-\mathrm{C}$ ), while upregulated genes were enriched for genes involved in steroid hormone biosynthesis and other metabolic pathways (Supplemental Table 1D). RNA-Seq gene expression data on thyroid glands from WT-LID and KO-LID mice analyzed with several pathway enrichment analysis programs revealed that pathways related to cell cycle, ECM receptor, cell adhesion, chemokine/cytokine pathways, 
A

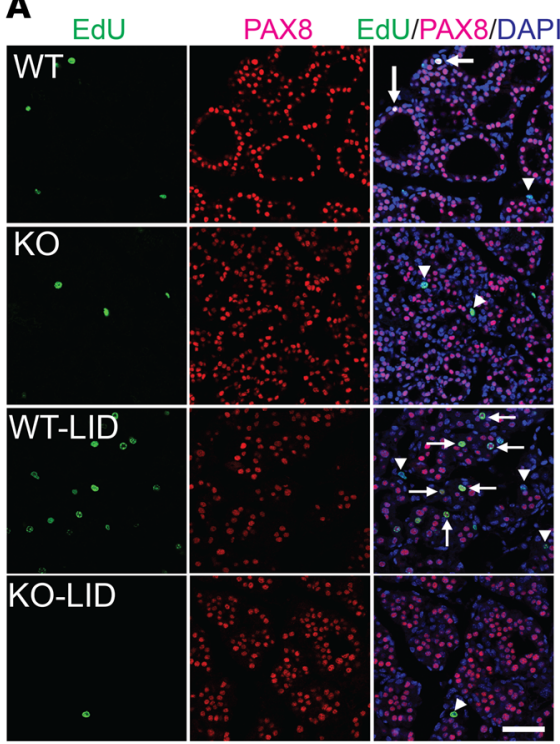

B

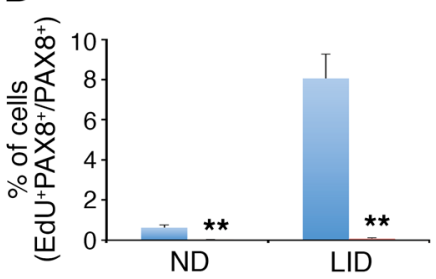

C
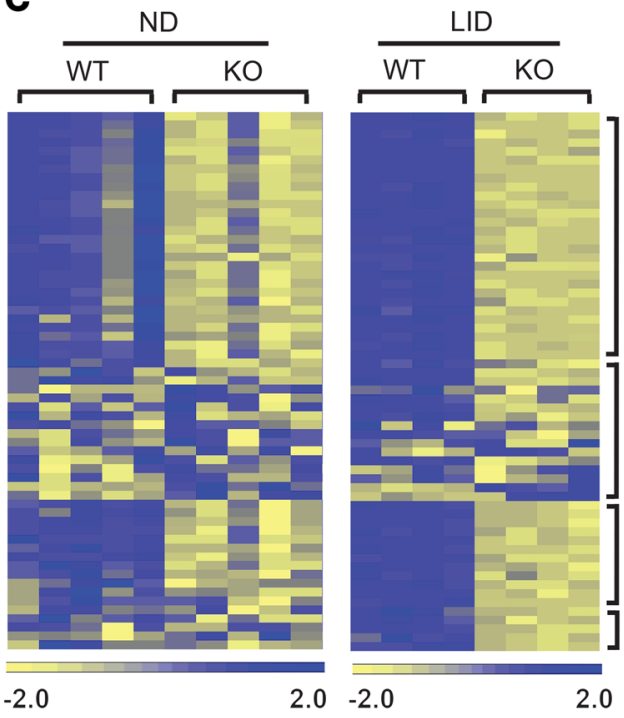

2.0

Cell cycle

TH biosynthesis

Extracellular

matrix

Cytokine/chemokine pathway
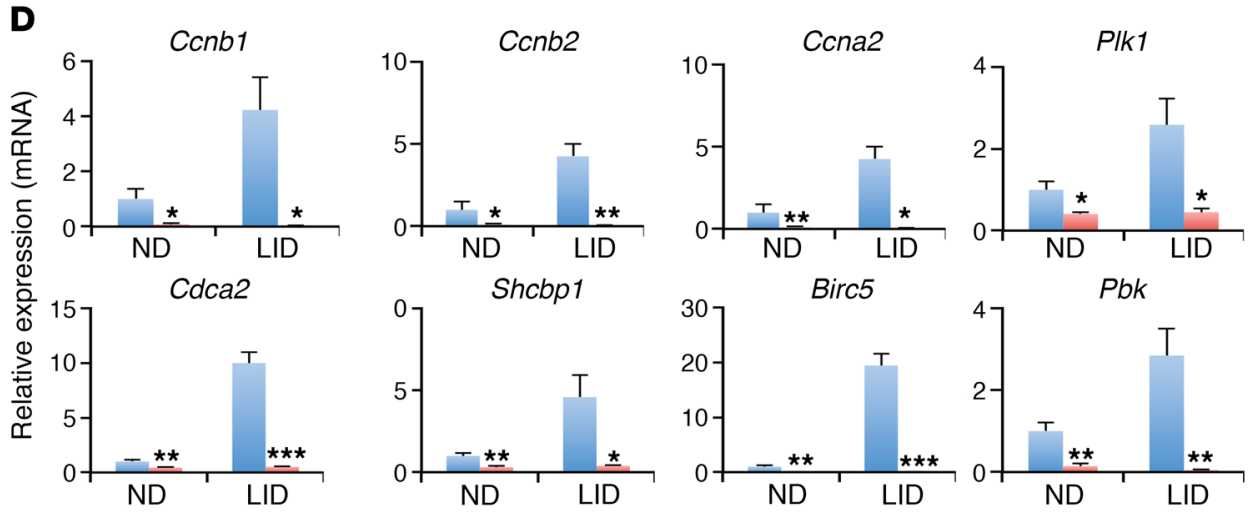

Figure 3. Proliferation of thyroid follicular cells was greatly reduced in KO-LID mice. (A) EdU incorporation was analyzed in the thyroid of 3-week-old WT and Clis3KO mice and WT-LID and KO-LID mice as described in Methods. Arrows indicate PAX8 ${ }^{+} \mathrm{EdU}^{+}$cells, and arrowheads indicate PAX8-EdU+ cells. Scale bar: $50 \mu \mathrm{m}$. (B) Percentages of PAX8 ${ }^{+}$cells staining EdU ${ }^{+}$were calculated and plotted. $n \geq 3$ for each group. (c) Heatmaps generated from gene expression profiles obtained by microarray analyses of thyroid glands from WT and Clis3KO mice fed either ND or LID as indicated. The same genes associated with cell cycle, TH biosynthesis, ECM, and cytokine/chemokine pathways as shown in Supplemental Table 3 are clustered as indicated. (D) QRT-PCR analysis of the expression of several cell proliferation regulatory genes in WT and Glis3KO mice fed with ND or LID. $n \geq 4$. Data are shown as mean \pm SEM. ${ }^{*} P<0.05$; ${ }^{* *} P<0.001$; ${ }^{* *} P<0.0001$, Student's $t$ test.

and $\mathrm{TH}$ synthesis were among the top biological processes regulated by GLIS3 (Figure 3C and Supplemental Table 2, A-D). Ccna2, Ccnb1 and -2, Ccnd1, Cdkn3, Mki67, E2f1, Cdca3, Birc5 (encoding survivin), Cdc25c, Plk1, Pbk, Shcbp1, and Bub1 were among the cell cycle control genes most downregulated in Glis3KO mice (Supplemental Table 3). Quantitative real-time PCR (QRT-PCR) analysis confirmed the reduced expression of many of these genes in thyroid glands from Glis3KO mice fed either a ND or a LID (Figure 3D).

In addition to cell cycle-regulatory genes, a number of ECMrelated genes, including several collagen genes, e.g., Col1a1 and Col3a1, were expressed at significantly lower levels in thyroid glands of Glis3KO mice compared with those of WT mice (Figure $3 \mathrm{C}$ and Supplemental Table 3). The expression of many of the cell cycle-regulatory and collagen genes was several-fold higher in thyroid glands from WT-LID mice compared with those receiv- ing ND, whereas their expression remained largely unchanged in KO-LID mice compared with Glis3KO. Thus, differences in the expression of cell cycle-regulatory and collagen genes between WT and Glis3KO thyroids were greatly amplified under LID conditions (Figure 3C, Supplemental Table 3, and Supplemental Figure 4). Similarly, a number of genes related to inflammatory and immune responses, including the chemo/cytokine genes $\mathrm{Ccl} 7$ and Ccl2, were also highly induced in thyroid glands from WT-LID mice compared with those from WT mice fed a ND, whereas these genes remained expressed poorly in KO-LID mice (Supplemental Table 3). These data suggest that, in addition to enhanced proliferation, thyroid glands from WT-LID mice display an increased fibrotic and inflammatory response and that these responses are suppressed in Glis3KO mice.

The repression of cell cycle-regulatory gene expression in the thyroid glands of Glis3KO mice is consistent with the observed 
A

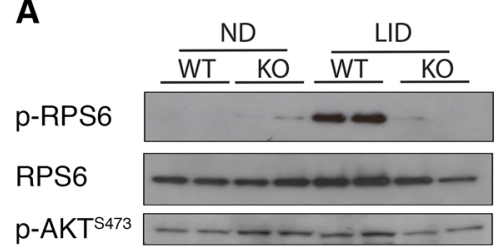

B

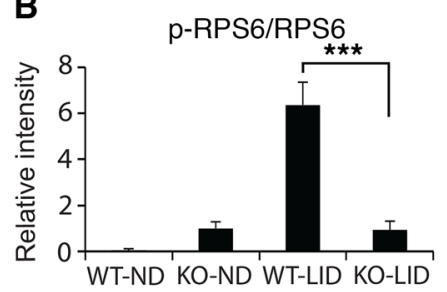

C
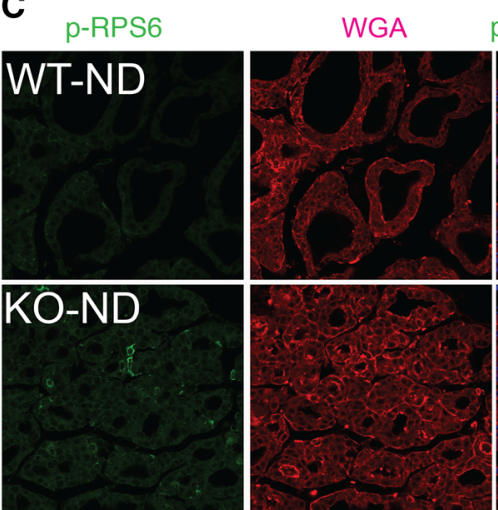

p-RPS6/WGA/DAPI
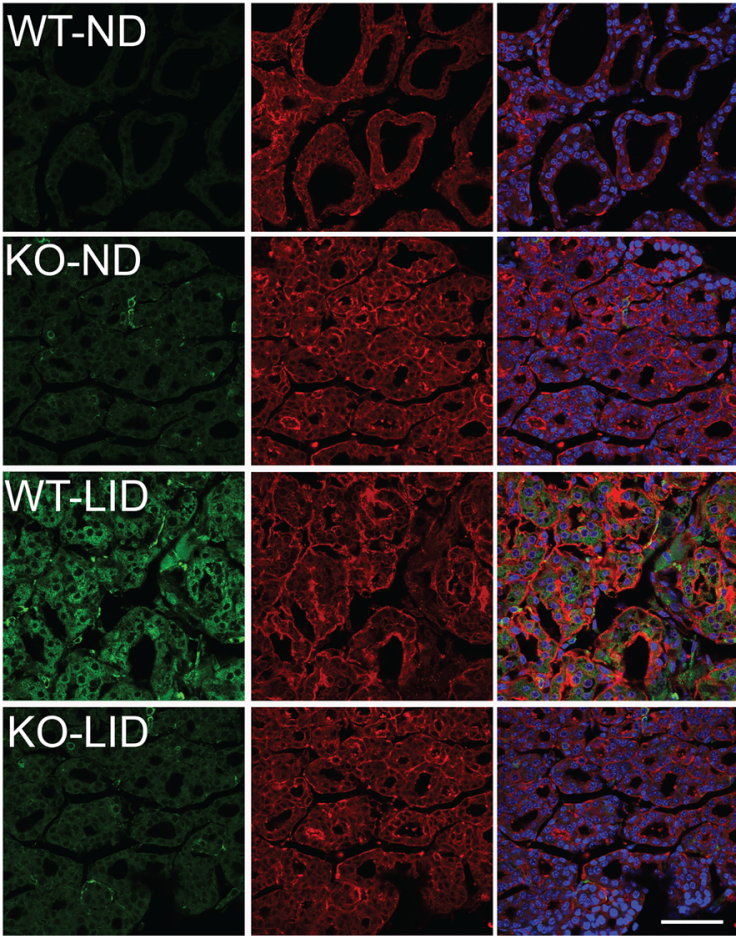

Figure 4. TSH/TSHR-mediated activation of the mTORC1 signaling pathway is impaired in the thyroid gland of KO-LID mice. (A) Protein lysates obtained from thyroid glands of WT and Glis3KO mice fed either ND or LID were examined by Western blot analysis using RPS6, p-RPS6, and $p-A K T^{5473}$ antibodies. (B) The relative levels of expression were analyzed and the ratio of p-RPS6/RPS6 plotted. $n=4$ for each group. Data are shown as mean $\pm \mathrm{SEM}$. ${ }^{* *} P<0.0001$, Student's $t$ test. (C) Representative images of sections of WT and Glis3KO thyroid tissue immunostained with antibodies against p-RPS6 (green), WGA (red), and DAPI. Scale bar: $50 \mu \mathrm{m}$.

decrease in EdU incorporation (Figure 3, A and B) and was further supported by the significant decrease in the number of cells staining positively for phosphorylated histone $\mathrm{H} 3(\mathrm{p}-\mathrm{H} 3), \mathrm{a} \mathrm{G}_{2} / \mathrm{M}$ phase cell cycle marker (Supplemental Figure 5). These data support the hypothesis that GLIS3 is essential for the proliferation of thyroid follicular cells and their response to TSH.

Activation of the mTORC1-signaling pathway is impaired in thyroid glands of Glis3KO mice. Previous studies have linked the LID- induced proliferation of thyroid follicular cells as well as thyroid follicular carcinoma cells to activation of the mTORC1 pathway (4, 41). To investigate whether activation of the mTORC1 pathway was affected in the thyroid glands of Glis3KO mice, we examined the phosphorylation of ribosomal protein S6, an mTORC1/RPS6K substrate. Consistent with previous reports, p-RPS6 levels were very low in thyroid glands from WT mice and dramatically increased when mice were fed a LID (Figure 4, A and B). In contrast, p-RPS6 did not increase and remained low in thyroid glands from KO-LID mice (Figure 4, A and B). These differences in p-RPS6 expression were supported by immunofluorescence staining (Figure 4C). These data suggest that GLIS3 is required for the activation of the mTORC1/RPS6K-signaling pathway. Interestingly, neither LID nor Glis3 deficiency changed the level of active, phosphorylated AKT1 $\left(\mathrm{p}-\mathrm{AKT1}^{\mathrm{S473}}\right)$ (Figure $\left.4 \mathrm{~A}\right)$, which can act upstream of mTORC1, indicating that the inhibition of RPS6 activation is independent of AKT1, consistent with a previous report (4). In addition, no difference in the activation of AMPK, which has been reported as inhibiting mTORC1 activation (42), or PKA was observed between WT and Glis3KO thyroid glands (Supplemental Figure 6).

GLIS3 is required for TH biosynthesis. It is well known that TSH induces the expression of many genes involved in TH biosynthesis, particularly the expression of $P d s$ and Nis and, to a lesser extent, that of Tpo and Duoxa2 $(6,7,9,10,43)$. Since TSH levels are greatly elevated in Glis3KO mice, one might surmise that these genes are expressed at significantly higher levels in Glis3KO compared with WT mice. However, despite the high TSH levels in Glis3KO mice, the expression of $P d s$ was reduced rather than increased (Supplemental Table 3, and Figure 5A), while the expression of Tpo was slightly elevated, that of the monocarboxylate transporter 8 ( $M c t 8$ also referred to as Slc16a2) moderately lower, and that of $\mathrm{Nis}, \mathrm{Tg}$, Duoxa2, Duoxa1, and Tshr not significantly altered. In WT-LID mice, the expression levels of $P d s, N i s$, and Tpo were substantially induced in response to greatly elevated serum TSH levels (Supplemental Table 3, and Figure 5A), consistent with previous reports (69), while Duoxa2 and Dio1 expression increased moderately (Figure 5A). In contrast, in Glis3KO mice with already elevated TSH levels, LID did not enhance the expression of Pds, Nis, Duoxa2, and Dio1 (Figure 5A). The expression levels of Pax8, TtfI (also referred to as $N k x 2.1$ ), and Ttf2 (FoxE1), which play an important role in thyroid gland development, were either slightly higher or not significantly changed in thyroid glands from Glis3KO mice (Figure 5, A and B and Supplemental Table 3) and little difference was observed in PAX8 and TTF1 protein expression (Figure 5B and Supplemental Figure 7). These findings support the conclusion that GLIS3 is essential for TH biosynthesis, consistent with our KEGG pathway analysis (Supplemental Table 1), and is not an absolute requirement for embryonic thyroid development. In contrast to the reduced expression of these genes, the expression of sulfotransferase 1E1 (Sult1E1), which catalyzes TH sulfonation (44), was increased 2-fold in Glis3KO and 7-fold in KO-LID thyroid glands compared with those of WT and WT-LID mice, respectively (Figure 5A).

Since the uptake of iodide through NIS is a critical first step in TH biosynthesis and because its regulation is well studied (9), we focused on the effect of GLIS3 on Nis expression in more detail. Western blot analysis and immunohistochemistry showed that NIS expression correlated with NIS protein levels. NIS protein levels 
A
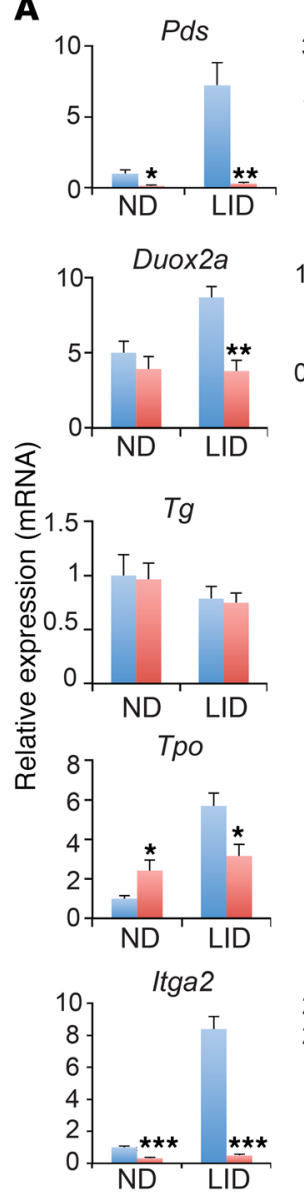
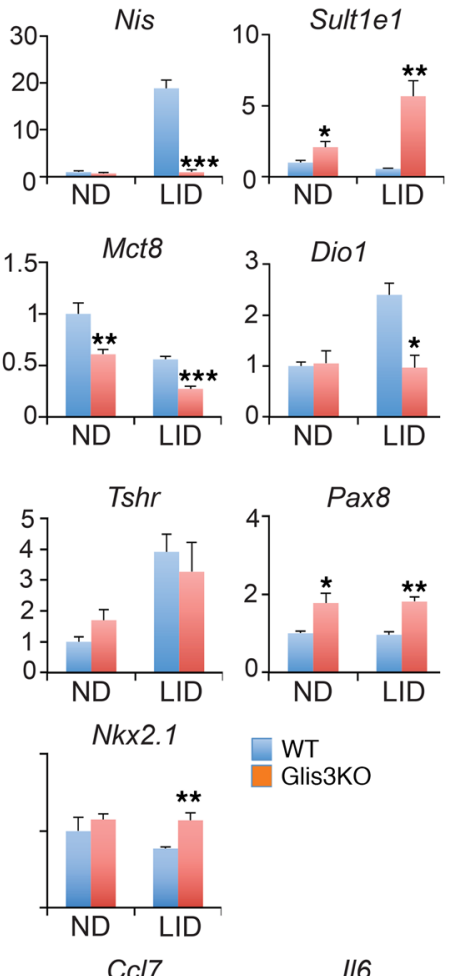

WT

Glis3KO
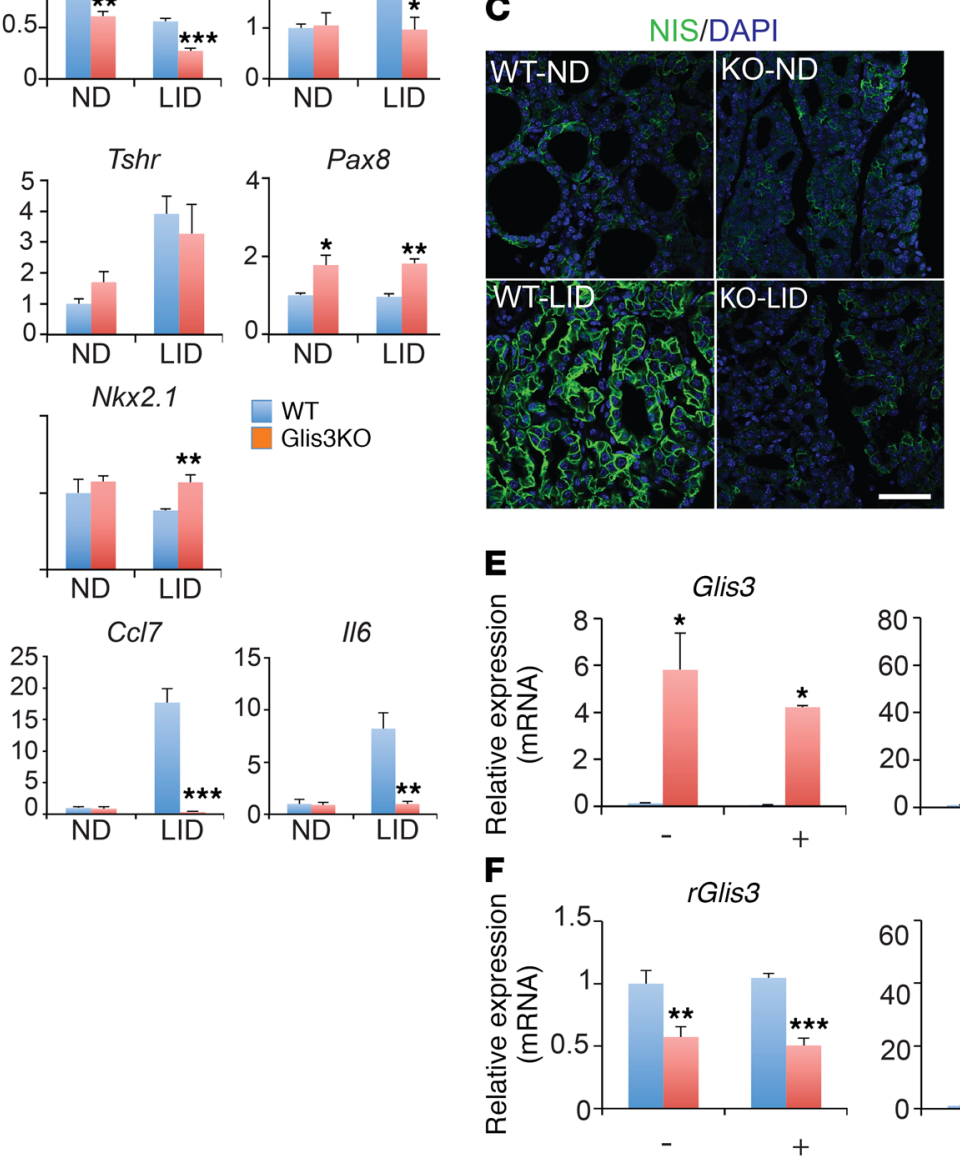

D
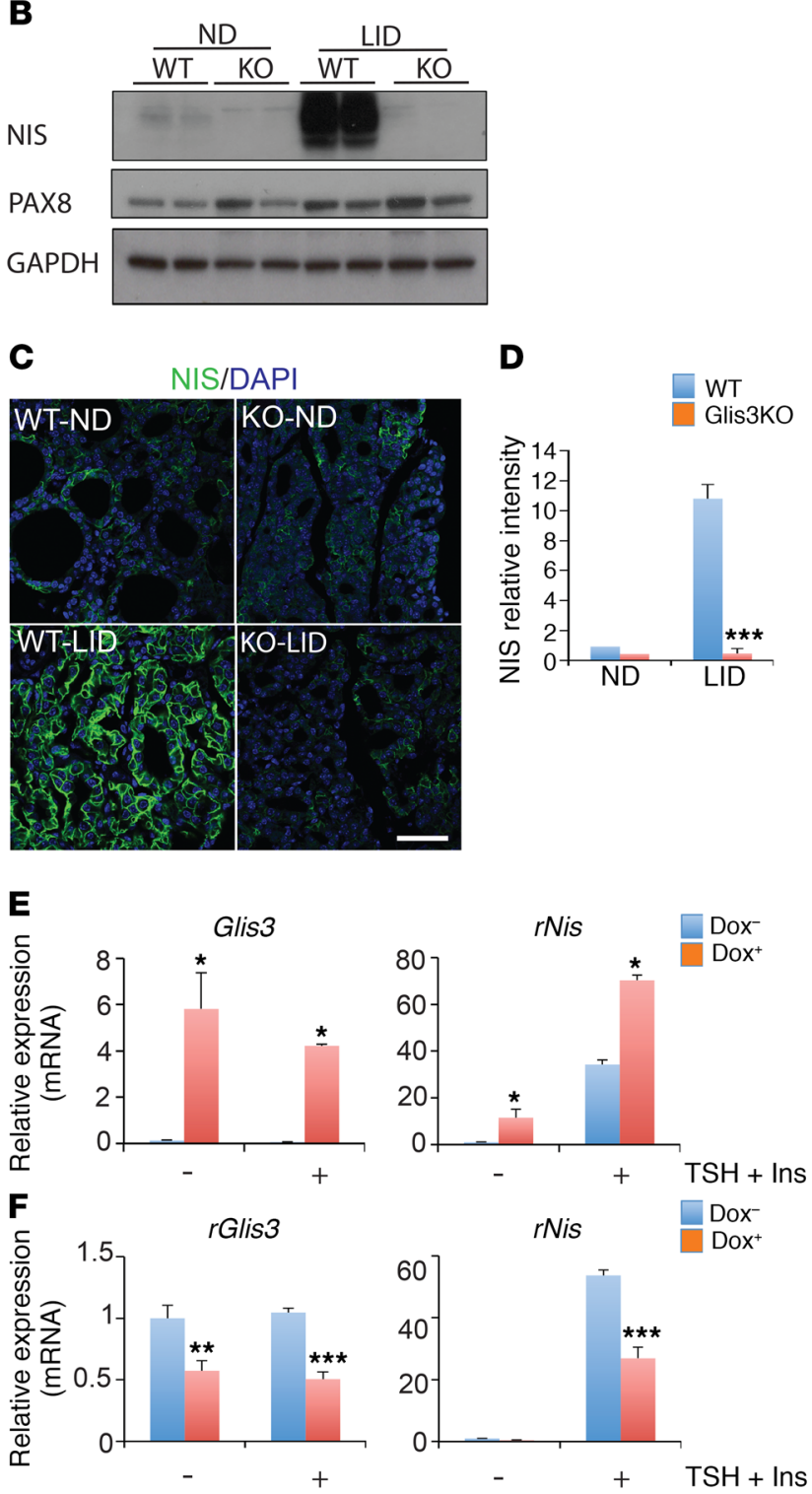

Figure 5. Loss of GLIS3 function decreases the expression of several genes required for TH biosynthesis. (A) Relative gene expression in thyroids from WT and Clis3KO mice fed either ND or LID was analyzed by QRT-PCR analysis. $n \geq 4$ for each group. (B) Protein lysates obtained from thyroids of WT and Glis3KO mice fed either ND or LID were examined by Western blot analysis with antibodies against NIS and PAX8. GAPDH was used as a loading control. (C) Representative images of sections of WT and Clis3KO thyroids immunoassayed with antibodies against NIS (green), WGA (red), and DAPI. Scale bar: $50 \mu \mathrm{m}$. (D) The relative intensity of NIS immunostaining was analyzed with ImageJ (NIH) and plotted. $n \geq 2$ for each group. (E) PCCI3-pIND20-Clis3 cells stably expressing Glis3 under the control of a Dox-inducible promoter were treated with or without Dox in the presence or absence of TSH and insulin (Ins). The expression of Clis3 and Nis was examined by QRT-PCR. (F) PCCI3 cells stably expressing Glis3 shRNA under the control of a Dox-inducible promoter to knock down Glis3 expression were treated with or without Dox in the presence or absence of TSH and insulin. The expression of Glis3 and Nis was examined by QRT-PCR. Data in $\mathbf{E}$ and $\mathbf{F}$ are derived from triplicate samples and representative of 2 independent experiments. Data are shown as mean \pm SEM. ${ }^{*} P<0.05 ;{ }^{* *} P<0.001 ;{ }^{* *} P<0.0001$, Student's $t$ test. rClis3 and rNis refer to rat genes.

were dramatically increased in the thyroid glands of WT-LID, but were not induced in Glis3KO or in KO-LID mice despite their high blood levels of TSH (Figure 5, A-D), suggesting a requirement for GLIS3 in NIS induction. To test this idea, we asked whether ectopic expression of GLIS3 would be sufficient to induce NIS expression in rat thyroid PCCl3 cells containing GLIS3 under the control of a doxycycline-inducible (Dox-inducible) promoter. Addition of Dox to these PCCl3 cells greatly induced GLIS3, which was accompanied by a 10-fold increase in Nis mRNA expression (Figure 5E). Dox-induced
GLIS3 in combination with TSH and insulin treatment, which have been reported to increase NIS expression (9), had a synergistic effect on NIS induction. In contrast, knockdown of Glis 3 by Dox-inducible Glis3 shRNA in PCCl3 cells reduced the expression of Glis3 as well as that of Nis by about $50 \%$ (Figure $5 \mathrm{~F}$ ). Together, these data support the hypothesis that GLIS3 plays a critical role in the regulation of Nis expression and is required for its optimal induction by TSH.

GLIS3 directly regulates the transcription of genes critical for $T H$ biosynthesis. To obtain further insights into the mechanism under- 
A



B


C
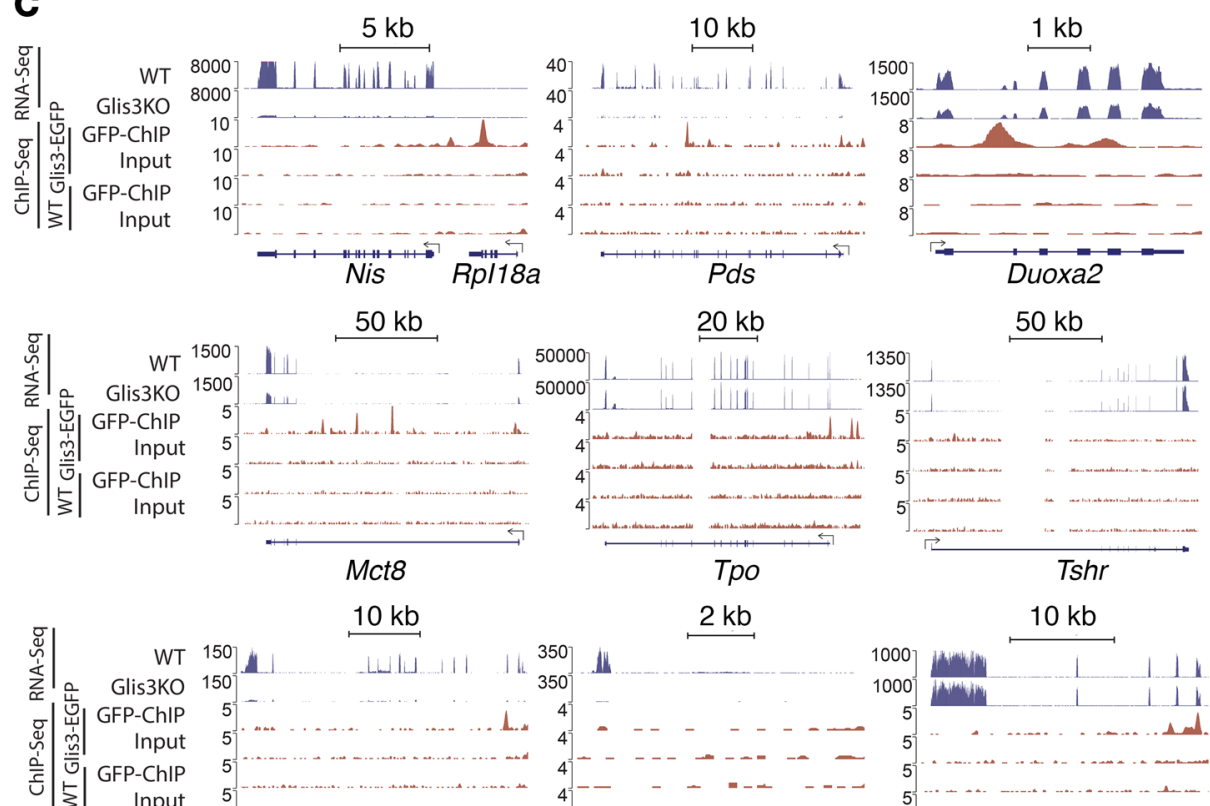

$10 \mathrm{~kb}$
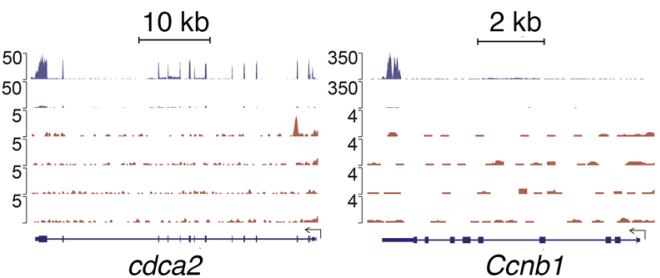

Figure 6. GLIS3 regulates the transcription of a subset of differentially expressed genes directly through its interaction with GLIS3-BS. (A) Pie chart showing the position of GLIS3-binding regions on the genome of the mouse thyroid gland relative to their nearest gene identified by Chipset analysis. The promoter is defined as the region up to $5 \mathrm{~kb}$ upstream of the TSS. (B) De novo consensus motif analysis using MEME program identified a G-rich GLIS3-binding motif, which is similar to GLIS3-BS identified previously by an in vitro binding assay. (C) GLIS3-EGFP occupancy on genome loci of several GLIS3-regulated genes associated with TH biosynthesis or cell proliferation. Tshr and Ccnb1 are included as negative controls. Gene tracks were taken from the UCSC Genome Browser (https://genome.ucsc.edu/). For each locus, Chipset analyses of input chromatin (Input) chromatin pulled down with GFPBP-agarose (GFP-ChIP) from GLIS3-EGFP and WT thyroid glands are shown as indicated. GLIS3-binding peaks were observed only in IP GFP. (D) Schematic view of the key regulatory functions of GLIS3 in thyroid follicular cells. TSH/TSHR activates several kinase pathways that subsequently lead to increased transcription of many genes required for TH biosynthesis. GLIS3 is essential for TH biosynthesis, acting by directly regulating the transcription of several key TH biosynthetic genes, particularly Pds and Nis. Prolonged elevated levels of TSH induce thyroid follicular cell proliferation and goiter development through AKT1-independent activation of the mTORC1 signaling pathway. GLIS3 is required for the activation of the mTORC1 pathway and thyroid follicular cell proliferation. GLIS3 is critical in mediating the downstream actions of TSH/TSHR. GLIS3 activity might be controlled by TSH/TSHR and regulated by one of the TSH/TSHR-activated kinase pathways.

lying the GLIS3-mediated regulation of the proliferation- and TH biosynthesis-related genes, we performed ChIP-Seq analysis of endogenous GLIS3 in thyroid glands from GLIS3-EGFP mice fed a LID for 2 weeks $(31,33)$. The genome-wide analysis of direct transcriptional targets (cistrome) of GLIS3 revealed enrichment for GLIS3 binding within and immediately upstream of 3,340 genes (Supplemental Table 4A). About 10\% of GLIS3 binding was within regions $5 \mathrm{~kb}$ upstream of transcription start sites (TSSs) (Figure 6A). De novo motif analysis of GLIS3-binding sites (GLIS3-BS) identified a consensus sequence motif (Figure 6B and Supplemental Figure 8) similar to the G-rich consensus GLIS3-BS identified previously by an in vitro binding assay (45). GO and pathway analy- sis showed that genes related to transcriptional regulation, transport, cell proliferation, and TH generation, which included Nis, Pds, Duoxa2, Tpo, Mct8, and Duox2, were among the top categories enriched for GLIS3 binding (Supplemental Table 4, B-E).

Comparison of the genes identified by ChIP-Seq or RNA-Seq analysis revealed that about $15 \%$ of the GLIS3 candidate target genes were differentially expressed between the thyroid glands from WT-LID and KO-LID mice (Supplemental Figure 9 and Supplemental Table 5, A and B). About $14 \%$ of the downregulated and $12 \%$ of the upregulated genes were directly regulated by GLIS3, consistent with our hypothesis that GLIS3 can function as a transcriptional activator as well as a repressor (28). GO 
analysis identified transport-related genes that included Nis and $P d s$ as among the top categories of downregulated genes directly regulated by GLIS3 (Supplemental Table 5C), whereas no highly significant correlation was found between a specific pathway and upregulated genes. GLIS3-EGFP occupancy on genome loci of several GLIS3-regulated genes is shown in Figure 6C. In addition to Nis and Pds, GLIS3 was also associated with Tpo, Dio1, Duoxa2, and $M c t 8$, the expression of which was only modestly affected by GLIS3 deficiency (Figure 6C and Supplemental Table 3), suggesting that they are direct transcriptional targets of GLIS3. Further analysis of GLIS3 occupancy at the Nis genome showed that GLIS3 enrichment was observed at regions $-1 \mathrm{~kb}$ and $-2.8 \mathrm{~kb}$ upstream of the Nis TSS, which was confirmed by ChIP analysis (Supplemental Figure 10). Interestingly, the $-2.8 \mathrm{~kb}$ region of the mouse Nis promoter corresponds to the rat Nis upstream enhancer (NUE)(46), which was reported to also bind PAX8 (Supplemental Figure 10, B and C). NUE-dependent reporter assays demonstrated that both GLIS3 and PAX8 were able to activate the NUE promoter and had an additive effect when expressed together (Supplemental Figure 11). Although both GLIS3 and PAX8 bind the NUE promoter, immunoprecipitation assays showed that GLIS3 and PAX8 did not interact and that GLIS3 also did not interact with TTF1 (Supplemental Figure 12). Our data indicate that GLIS3 controls TH biosynthesis by directly regulating the transcription of several key TH biosynthesis genes, particularly Nis and Pds. GLIS3 occupancy was also observed at several cell proliferation-related genes, such as Ccnd2 and Cdca2 (Figure 6C), but not at Ccnb1, Ccnb2, Ccna2, $C d k n 3, E 2 f 1$, and Bub1, genes that were the most downregulated in Glis3KO mice (Supplemental Table 3). GLIS3 was also found to be associated with a few ECM and inflammatory response genes (Supplemental Table 5C), but not the ones that were the most downregulated in Glis3-KO mice (Supplemental Table 3). These data suggest a largely indirect role for GLIS3 in the transcriptional regulation of ECM- and inflammatory/immune-related genes.

\section{Discussion}

GLIS3 deficiency in humans is associated with the development of congenital hypothyroidism; however, the underlying mechanism is poorly understood (24-28). In this study, we show that loss of GLIS3 function in mice also leads to the development of congenital hypothyroidism, as indicated by the significant decrease in serum TH concentration and greatly elevated serum TSH levels (Figure 1, A and B). Although in the first week after birth, the average size of the thyroid follicles in Glis3KO mice is smaller, thyroid morphology and serum $\mathrm{TH}$ levels are not significantly different from those in WT mice (Figure 2A and Supplemental Figure 3). This, together with our data showing that the expression of genes critical for thyroid development, including Pax8, Ttf1 (Nkx2.1), and Ttf2 (FoxE1), was either slightly increased or unchanged in thyroid glands of Glis3KO mice (Supplemental Table 3, Figure 5, A and B, and Supplemental Figure 7), suggests that the development of congenital hypothyroidism in Glis3KO mice is not related to thyroid gland dysgenesis. Instead, GLIS3 is essential for TH biosynthesis and postnatal thyroid gland development. Therefore, the development of hypothyroidism in Glis3KO mice appears to be related to dyshormonogenesis. In patients with mutations in GLIS3, the congenital thyroid phenotype varies from aplasia/ dysplasia to dyshormonogenesis, suggesting that genetic background or the nature of the mutation may influence the extent of the GLIS3 thyroid phenotype (37). Therefore, it is possible that in a different genetic background, GLIS3 deficiency might also affect embryonic thyroid development.

$\mathrm{TSH}$, through its receptor and the subsequent activation of several protein kinases, including PKA, PKC, AMPK, and PI3K/ mTOR1, positively regulates the expression of a number of genes critical for TH biosynthesis, including Pds, Nis, and Tpo (6, 7, 9, 10). Despite the high level of TSH in Glis3KO mice, the expression of several of these genes, particularly $P d s$ and Nis, was not induced. Under LID conditions, serum TSH levels were equally highly elevated in both WT and Glis3KO mice (Figure 2G); however, $P d s$ and Nis expression remained low in Glis3KO mice, while they were, as expected, highly induced in WT mice (Figure 5A). Similarly, Tpo, Dio1, and Duoxa2 were moderately induced in WT-LID mice and also expressed at lower levels in thyroid glands of KO-LID mice. The expression of Tshr was not significantly changed in thyroid glands of Glis3KO mice, suggesting that the decreased expression of $P d s$ and Nis is not due to the reduction in $T s h r$ expression. These observations indicate that GLIS3 is required for the TSH/TSHR-mediated induction of several genes critical for TH biosynthesis. Genetic variants and mutations in Nis, Pds, Tpo, and Duoxa2 have been linked to hypothyroidism $(9,20-23,40)$; the observed decrease in the expression of these genes is therefore, at least in part, responsible for the development of hypothyroidism in Glis3KO mice and would explain the development of congenital hypothyroidism in the group of GLIS3 patients with dyshormonogenesis $(24-28,37)$.

The mechanisms that regulate the transcription of Nis and $P d s$ gene expression are not yet fully understood. Nis expression is under complex control and regulated at both the transcriptional and posttranscriptional levels $(9,47)$. It is believed that Nis transcription is regulated by the TSH/TSHR-mediated activation of the PKA pathway $(9,48)$. Another study indicated that PAX8 regulates Nis transcription by binding directly to the NUE promoter region, which is critical for Nis transcriptional regulation (46), while TTF1 has been reported to play a role in the regulation of $P d s$ transcription (49). ChIP-Seq analysis revealed that GLIS3 is associated with several TH biosynthetic genes that are suppressed in the thyroid gland of Glis3KO mice, including $P d s$ and Nis, indicating that their transcription is directly regulated by GLIS3 (Figure 6C and Supplemental Table 3). This was further supported by the observed enrichment of GLIS3 at the NUE region (Supplemental Figure 10) (46) and by the ability of GLIS3 to activate this promoter region (Supplemental Figure 11). Although ChIP-Seq identified $\operatorname{Pax} 8$ as a direct transcriptional target of GLIS3, Pax 8 expression was slightly enhanced rather than decreased in Glis3KO mice, indicating that Nis repression in these mice was unrelated to changes in Pax 8 expression. Although GLIS3 and PAX8 cooperated in the optimal activation of the NUE promoter, these 2 proteins do not appear to interact (Supplemental Figure 11 and Supplemental Figure 12). Future studies need to determine in what ways these 2 transcription factors cooperate in regulating Nis transcription. Together, our data suggest that the decrease in Nis and Pds expression in Glis3KO mice is due to loss of their transcriptional activation by GLIS3. 
In addition to the critical function of GLIS3 in TH biosynthesis, our study demonstrates that it also has a key role in the regulation of thyroid follicular cell proliferation. The relative decrease in the number of follicular cells/follicle was not due to increased apoptosis or autophagy, but was related to reduced proliferation of thyroid follicular cells in Glis3KO mice, as indicated by the decrease in $\mathrm{PAX} 8^{+} \mathrm{EdU} \mathrm{U}^{+}$and $\mathrm{p}-\mathrm{H} 3^{+}$cells (Figure 3, $A$ and B and Supplemental Figure 5). The reduced proliferation of thyroid follicular cells is supported by gene profiling analysis showing decreased expression of a number of key cell cycleregulatory genes (Supplemental Table 3 and Figure 3D). Chronic elevation of TSH, as in LID, induces thyroid follicular cell proliferation and promotes goiter development, as observed in our WT mice (4). However, in Glis3KO mice, in which TSH is greatly elevated, TSH-mediated induction of cell proliferation and cell cycle-regulatory genes and goiter development were not observed. The difference in thyroid follicular cell proliferation between thyroid glands of WT and Glis3KO mice became even more distinct in mice fed a LID (Supplemental Table 3 and Figure 3, C and D), and differences in the expression of cell cycleregulatory genes between WT and Glis3KO mice were magnified under these conditions. Although ChIP-Seq analysis showed that a few proliferation-related genes were directly regulated by GLIS3 (Supplemental Table 5C), the cell cycle-regulatory genes most affected by GLIS3 deficiency, including Ccnb1/2, Mki67, $C d c a 3$, and Ccna2, were not directly targeted by GLIS3 (Supplemental Table 3), suggesting that transcriptional regulation of these genes by GLIS3 is perhaps indirect. In addition to its effect on cell cycle genes, the loss of GLIS3 function abrogates the induction of fibrosis and inflammatory genes in KO-LID mice. As they are proliferation-regulatory genes, though a few direct target genes were identified by ChIP-Seq analysis, the regulation of these genes by GLIS3 is largely mediated by an indirect mechanism (Figure 6D and Supplemental Table 3).

The mTORC1 pathway plays a critical role in promoting proliferation in many cell systems and has been implicated in several cancers, including follicular thyroid cancer $(50,51)$. The increased proliferation observed during chronic activation of the TSHR by TSH has been linked to AKT1-independent activation of the mTORC1 pathway (4). We showed that the loss of GLIS3 function abrogates the TSH/TSHR-mediated activation of the MTORC1/ RPS6K signaling pathway in KO-LID mice, suggesting that GLIS3 is required for the activation of this pathway in thyroid follicular cells (Figure 4A). Consequently, the lack of mTORC1 activation explains, at least in part, the loss of the proliferative response in Glis3KO thyroid follicular cells to elevated TSH. Since levels of p-AMPK, an inhibitor of mTORC1, did not change in Glis3KO thyroid glands, the inhibition of the MTORC1 pathway was not related to AMPK. The amino acid transporter SLC7A7 has been reported as positively regulating the mTORC1 pathway $(52,53)$. The expression of Slc7a7 mRNA was substantially decreased in thyroid of Glis3KO compared with that of WT (data not shown) and might contribute to reduced activation of the MTORC1 pathway. Further studies are needed for understanding the link between TSH and the activation of the mTORC1 pathway and its regulation by GLIS3.

Collectively, our studies show that the loss of GLIS3 function inhibits cell proliferation of thyroid follicular cells as well as TH biosynthesis, both of which are regulated by the TSH/TSHR pathway. These observations support a model wherein GLIS3 is a key mediator acting downstream of TSH/TSHR (Figure 6D). Several kinase pathways activated by TSH/TSHR have been implicated in mediating the downstream effects of TSH on gene expression, proliferation, and TH biosynthesis. We previously reported that GLIS3 undergoes a number of posttranslational modifications, including phosphorylation and ubiquitination, that can regulate GLIS3 transcriptional activity $(54,55)$. This raised the possibility of a connection between TSH/TSHR-activated kinase pathways and the regulation of GLIS3 activity. Moreover, the recurrent positive- and negative-feedback regulation of TH biosynthesis may require tight regulation of GLIS3 transcription activity. We propose that activation of a particular kinase by TSH/TSHR leads to GLIS3 phosphorylation, enhances GLIS3 transcriptional activity, and induces transcription of TH biosynthetic genes, which subsequently results in increased TH biosynthesis (Figure 6D). Similarly, such posttranslational modifications of GLIS3 might also play a role in its regulation of the mTORC1 pathway and, consequently, the expression of several cell cycle genes and thyroid follicular cell proliferation.

In summary, in this study, we demonstrate that GLIS3 is essential for TSH/TSHR-mediated TH biosynthesis and thyroid follicular cell proliferation. These functions also provide a mechanism explaining why GLIS3 deficiency leads to neonatal hypothyroidism and prevents the development of goiter. Elucidation of upstream pathways that regulate the transcriptional activity of GLIS3 may lead to new therapeutic strategies in the management of some thyroid gland-associated pathologies.

\section{Methods}

Mice. The Glis3-EGFP mice (C57BL/6-Glis3 < tm3(Glis3-EGFP) Amj>), which express a GLIS3-EGFP fusion protein to monitor GLIS3 expression, and the Glis3-deficient (Glis3KO; C57BL/6-Glis3 $<\operatorname{tm} 3$ (mCherry)Amj>) mice were described previously $(31,33)$. Both mouse lines were backcrossed onto C57BL/6 for at least 6 generations. Mice were routinely fed a NIH-31 diet (ND; Harlan). For the LID study, mice were fed with a TD.95125 diet (Harlan) for 6 days right after weaning.

Measurement of serum and tissue TH levels. Serum T3, T4, and $\mathrm{TSH}$ levels and free and thyroglobulin-bound $\mathrm{T} 3$ and $\mathrm{T} 4$ contents in thyroid gland were measured by radioimmunoassay as described in detail previously $(40,56)$. T4 was also analyzed by ELISA according to the manufacturer's instructions (Calbiotech). Serum TSH was also measured by MILLIPLEX MAP Rat Thyroid Magnetic Bead Panel (Millipore).

Histology and immunohistochemistry. For histological analysis, thyroid glands were fixed overnight in 10\% neutralized buffered formalin (NBF) or Bouin's solution, washed with $1 \times$ PBS, and embedded in paraffin. Sections $(5 \mu \mathrm{m})$ were then stained with H\&E. For immunohistochemistry, thyroid glands were fixed overnight in $4 \%$ paraformaldehyde, washed with $1 \times$ PBS, and then incubated in $30 \%$ sucrose for cryoprotection. The tissues were subsequently embedded and frozen in OCT medium (Tissue-Tek). Frozen sections $(10 \mu \mathrm{m})$ obtained with a cryostat (Leica) were placed on slides, dried at room temperature, washed with Tris-buffered saline with 0.02\% tween-20 (TBST), and then incubated overnight at $4^{\circ} \mathrm{C}$ with antibodies against GFP (1:500; 
Aves Labs), PAX8 (1:300; Novus Biologicals, catalog NBP1-32440), RPS6 (1:300; Cell Signaling Technology, catalog 2217), p-RPS6 (1:300; Cell Signaling Technology, catalog 2211), p-H3 (1:300; Abcam, Ab1576), TTF1 (1:300; Abcam, ab76013), or PECAM1 (1:300; BD Pharmingen, catalog 550274) as indicated. The anti-NIS antibody was provided by N. Carrasco (Yale University, New Haven, Connecticut, USA). Autophagic cells were examined by immunohistochemistry with an antibody against LC3A/B (1:300; Cell Signaling Technology, cata$\log 12741)$ (57). Anti-chicken or anti-rabbit Alexa Fluor 488- or Alexa Fluor 594-conjugated antibodies (1:1000, Life Technologies) were used as secondary antibodies. Alexa Fluor 647-conjugated wheat germ agglutinin (WGA) (1:100, Thermo Fisher Scientific) was used for selective staining of cell membrane. Apoptotic cells were examined using an In Situ Cell Death Detection Fluorescein Kit (Roche) following the manufacturer's instructions. Fluorescence was observed with a Zeiss LSM780 confocal microscope.

EdU incorporation assay. Proliferation of thyroid follicular cells was analyzed by the EdU incorporation assay. Thyroid glands from 3-week-old WT and Glis3KO mice fed either ND or LID were isolated 1.5 hours after peritoneal injection of EdU $(25 \mathrm{mg} / \mathrm{kg})$ and then prepared for frozen sections as described above. EdU was detected with a Click-iT Plus EdU Alexa Fluor 488 Imaging Kit (Thermo Fisher Scientific) following the manufacturer's instructions. Thyroid follicular cells were identified by anti-PAX8 antibody staining. To calculate the number of $\mathrm{EdU}^{+} \mathrm{PAX} 8^{+}$cells, more than 2,000 $\mathrm{PAX} 8^{+}$cells were counted in each thyroid $(n=3-5)$.

Microarray analysis. Thyroid glands were collected from 1-month-old WT $(n=5)$ and Glis3KO $(n=5)$ mice fed a ND and 4 WT and 4 Glis3KO mice fed a LID for 6 days. RNA was extracted using a RNAqueous-Micro Kit (Thermo Fisher Scientific) according to the manufacturer's instructions, amplified, and labeled Cy3 as described in the NuGEN Ovation Pico WTA System protocol (NuGEN) for Agilent microarrays. Gene expression analysis was carried out using Agilent Whole Mouse Genome $4 \times 44$ Multiplex Format Oligo Arrays (Agilent Technologies) as described previously (31). Data obtained with the Agilent Feature Extraction software (v12) were further analyzed with OmicSoft Array Studio (version 7.0).

RNA-Seq analysis. Thyroids from WT $(n=3)$ and Glis3KO $(n=3)$ mice fed a LID were collected. RNA was extracted and amplified using a TruSeq RNA Library preparation kit. Sequencing reads were obtained using a MiSeq Sequencing System (Illumina). High-quality reads were aligned to the mouse genome (assembly: mm9) using RNA-STAR aligner and reads mapping to each gene were calculated using the HTSeq Toolkit. Differential gene expression analysis was carried out through edgeR package. Genes with a minimum of 2 -fold expression difference and FDR of less than 0.01 were considered as differentially expressed. Pathway analyses were performed using databases including KEGG, Reactome (http://reactome.org/), Biocarta from David Bioinformatics Resources (http://david.abcc.ncifcrf.gov/), ConsensusPathDB (http://cpdb.molgen.mpg.de/MCPDB), and ToppGene (https:// toppgene.cchmc.org/). Analysis of the GO biological process was performed with David Bioinformatics Resources.

QRT-PCR analysis. RNA was extracted from thyroid glands or PCCl3 cells and cDNA generated using a High Capacity cDNA Reverse Transcription Kit (Applied Biosystems). QRT-PCR was performed in triplicate in a StepOnePlus Real Time PCR System (Applied Bio- systems). Results were normalized to an $18 \mathrm{~S}$ internal control. Primer sequences are listed in Supplemental Table 6.

Western blot analysis. Mouse thyroid glands were homogenized with a micropestle in $1 \times$ RIPA buffer (EMD Millipore) supplemented with a protease and phosphatase inhibitor cocktail (PIC) (Thermo Fisher Scientific). The homogenates were centrifuged and proteins in the soluble fraction examined by Western blot analysis with antibodies against RPS6, p-RPS6, p-AKT (S473) (p-AKT ${ }^{\mathrm{s} 73}$; catalog 9271), p-AMPK $\alpha$ (p-AMPK ${ }^{\mathrm{T} 172}$, catalog 2535), p-PKA (p-PKA ${ }^{\mathrm{T} 197}$, catalog 4781) (all from Cell Signaling Technology); PAX8, GAPDH (Santa Cruz Biotechnology Inc., catalog SC-25778), TTF1, HA (catalog 11867423001, MiliporeSigma), and NIS. HRP-conjugated secondary antibodies were used for detection. Signal intensities were analyzed by a gel imaging system (Alpha Innotech).

Overexpression and knockdown of Glis3 in PCCl3 cells. Rat thyroid follicular PCCl3 cells (ATCC CRL-1468) were cultured in Coon's/F12 media supplemented with $5 \%$ FBS, $1 \mathrm{~m} \mathrm{IU} / \mathrm{ml} \mathrm{TSH}$, $10 \mathrm{mg} / \mathrm{ml}$ insulin, $5 \mathrm{mg} / \mathrm{ml}$ apo-transferrin, and $10 \mathrm{nM}$ hydrocortisone. PCCl3-pIND20-Glis3 cells were generated by infection of pIND20-Glis3 lentivirus containing Dox-inducible GLIS3 tagged with Flag at its $\mathrm{N}$ terminus. Dox-inducible Glis3 knockdown in PCCl3 cells was performed by infection of pZIP-TRE3G-shGlis3 or pZIP-TRE3G-scrambled (TransOMIC Technology). Expression was induced with $300 \mathrm{ng} / \mathrm{ml}$ Dox.

ChIP. To identify genes directly regulated by GLIS3, we performed ChIP-Seq analysis. Thyroid glands from WT and Glis3-EGFP mice fed a LID were isolated and homogenized in PBS containing a protease and PIC (Thermo Scientific) with a Tekmar Tissumizer (Tekmar Co.) and then fixed in $1 \%$ formaldehyde on ice for 10 minutes and subsequently for 10 minutes at room temperature. After a wash in PBS and lysis in IP Lysis Buffer (Thermo Fisher Scientific, catalog 87788) containing PIC, the cross-linked chromatin was sonicated for 40 minutes (S220 focused ultrasonicator, Covaris) and then incubated overnight with agarose crosslinked with a GFP-binding peptide (GFP-BP) (R. Petrovich, NIEHS) to pull down GLIS3-EGFP-chromatin complexes. Thyroid glands from WT mice served as negative controls to determine specificity. After subsequent washes, ChIPed-DNA was eluted as reported previously (58) and amplified using a TruSeq RNA Library Prep Kit. Sequencing reads were obtained using a MiSeq Sequencing System (Illumina). For binding site definition (peak calls), aligned reads were processed using SISSRs using default settings (59). Genes with promoters within a $5 \mathrm{~kb}$ distance from GLIS3 peaks were considered for further analyses.

NUE cloning and reporter assay. The NUE region between -3009 bp and -2550 bp upstream of the Nis promoter was amplified with primers GCGGTACCCTACAGAATGTGGGGGCATT and GCGAGCTCAGCAGGGCTAGCATGTTGTT and inserted into the KpnI and SacI restriction sites of pTAL-Luc reporter plasmid. Reporter assay was performed using HEK293T cells as described in our previous report (33).

Coimmunoprecipitation. The expression vectors p-3'FLAGCMV-Glis3-HA, p-3'FLAG-CMV-Glis3 4 N496-HA, p-3'FLAGCMV-Glis3 $\triangle$ C480-HA, and pCMV-SUFU-Myc were described previously $(54,55)$. pcDNA3.1-Pax8 was obtained from GenScript, and pcDNA3.1-TTF1 was a gift from David Mu (Pennsylvania State University, Hershey, PA, USA) (Addgene plasmid, catalog 49989). HEK293T cells (ATCC CRL-3216) were transfected with pcDNA3.1-Ttf1 and pcDNA3.1-Pax8, and 24 hours after transfection, cells were lysed in $1 \times$ RIPA buffer. Cell lysate was incubated with HA antibody and Dyna- 
beads Protein G (ThermoFisher) and then washed 2 times with RIPA buffer and 3 times with 1 TBS. Protein complexes were solubilized in NuPage LDS sample buffer (Thermo Fisher) and visualized by Western blot analysis.

Accession numbers. All original microarray data were deposited in the NCBI's Gene Expression Omnibus database (GEO GSE86841; ChIP-Seq and RNA-Seq data, GSE103297).

Statistics. Data were analyzed using 2-tailed Student's $t$ test, as indicated in the legends. Data were shown as mean \pm SEM. $P<0.05$ was considered significant.

Study approval. All animal studies followed guidelines outlined by the NIH Guide for the Care and Use of Laboratory Animals (National Academies Press, 2011). Protocols were approved by the IACUC at the NIEHS.

\section{Author contributions}

HSK and AMJ designed experiments and wrote the manuscript. HSK, GL, KLK, and XHL conducted experiments. HSK, AMJ, KG, DK, RJ and SR analyzed data.

\section{Acknowledgments}

This research was supported by the Intramural Research Program of the NIEHS, the NIH Z01-ES-100485 (to AMJ), Z01ES102625 (to RJ), and R37DK15070 from NIDDK, NIH (to SR).

Address correspondence to: Anton M. Jetten, NIEHS, NIH, 111 T.W. Alexander Drive, Research Triangle Park, North Carolina 27709, USA. Phone: 919.541.2768; Email: jetten@niehs.nih.gov.
1. Mondal S, Raja K, Schweizer U, Mugesh G. Chemistry and biology in the biosynthesis and action of thyroid hormones. Angew Chem Int Ed Engl. 2016;55(27):7606-7630.

2. Kleinau G, Neumann S, Grüters A, Krude H, Biebermann H. Novel insights on thyroidstimulating hormone receptor signal transduction. Endocr Rev. 2013;34(5):691-724.

3. Bagriacik EU, Yaman M, Haznedar R, Sucak G, Delibasi T. TSH-induced gene expression involves regulation of self-renewal and differentiation-related genes in human bone marrow-derived mesenchymal stem cells. J Endocrinol. 2012;212(2):169-178.

4. Brewer C, Yeager N, Di Cristofano A. Thyroidstimulating hormone initiated proliferative signals converge in vivo on the mTOR kinase without activating AKT. Cancer Res. 2007;67(17):8002-8006.

5. Dremier S, et al. The role of cyclic AMP and its effect on protein kinase $A$ in the mitogenic action of thyrotropin on the thyroid cell. Ann N Y Acad Sci. 2002;968:106-121.

6. Kero J, et al. Thyrocyte-specific Gq/G11 deficiency impairs thyroid function and prevents goiter development. J Clin Invest. 2007;117(9):2399-2407.

7. Postiglione MP, et al. Role of the thyroidstimulating hormone receptor signaling in development and differentiation of the thyroid gland. Proc Natl Acad Sci U S A. 2002;99(24):15462-15467.

8. Craps J, et al. Involvement of $\mathrm{mTOR}$ and regulation by AMPK in early iodine deficiency-induced thyroid microvascular activation. Endocrinology. 2016;157(6):2545-2559.

9. Dohán O, et al. The sodium/iodide Symporter (NIS): characterization, regulation, and medical significance. Endocr Rev. 2003;24(1):48-77.

10. Pesce L, et al. TSH regulates pendrin membrane abundance and enhances iodide efflux in thyroid cells. Endocrinology. 2012;153(1):512-521.

11. De Leo S, Lee SY, Braverman LE. Hyperthyroidism. Lancet. 2016;388(10047):906-918.

12. Grasberger H, Refetoff S. Genetic causes of congenital hypothyroidism due to dyshormonogenesis. Curr Opin Pediatr. 2011;23(4):421-428.

13. Wassner AJ, Brown RS. Congenital hypothyroidism: recent advances. Curr Opin Endocrinol Diabetes Obes. 2015;22(5):407-412.

14. Grüters A, Krude H. Detection and treatment of congenital hypothyroidism. Nat Rev Endocrinol. 2011;8(2):104-113.

15. Schoenmakers N, Alatzoglou KS, Chatterjee
VK, Dattani MT. Recent advances in central congenital hypothyroidism. J Endocrinol. 2015;227(3):R51-R71.

16. Stoupa A, Kariyawasam D, Carré A, Polak M. Update of Thyroid Developmental Genes. Endocrinol Metab Clin North Am. 2016;45(2):243-254.

17. Szinnai G. Clinical genetics of congenital hypothyroidism. Endocr Dev. 2014;26:60-78.

18. Rastogi MV, LaFranchi SH. Congenital hypothyroidism. Orphanet J Rare Dis. 2010;5:17.

19. Marians RC, Ng L, Blair HC, Unger P, Graves PN, Davies TF. Defining thyrotropin-dependent and -independent steps of thyroid hormone synthesis by using thyrotropin receptor-null mice. Proc Natl Acad Sci U S A. 2002;99(24):15776-15781.

20. Grasberger H, et al. Mice deficient in dual oxidase maturation factors are severely hypothyroid. Mol Endocrinol. 2012;26(3):481-492.

21. Fu C, et al. Mutation screening of the SLC26A4 gene in a cohort of 192 Chinese patients with congenital hypothyroidism. Arch Endocrinol Metab. 2016;60(4):323-327.

22. Kühnen $P$, et al. Identification of PENDRIN (SLC26A4) mutations in patients with congenital hypothyroidism and "apparent" thyroid dysgenesis. JClin Endocrinol Metab. 2014;99(1):E169-E176.

23. Nicholas AK, et al. Comprehensive screening of eight known causative genes in congenital hypothyroidism with gland-in-situ. J Clin Endocrinol Metab. 2016;101(12):4521-4531.

24. Dimitri P, et al. Expanding the clinical spectrum associated with GLIS3 mutations. JClin Endocrinol Metab. 2015;100(10):E1362-E1369.

25. Dimitri P, et al. Novel GLIS3 mutations demonstrate an extended multisystem phenotype. Eur J Endocrinol. 2011;164(3):437-443.

26. Habeb AM, et al. Incidence, genetics, and clinical phenotype of permanent neonatal diabetes mellitus in northwest Saudi Arabia. Pediatr Diabetes. 2012;13(6):499-505.

27. Senée V, et al. Mutations in GLIS3 are responsible for a rare syndrome with neonatal diabetes mellitus and congenital hypothyroidism. Nat Genet. 2006;38(6):682-687.

28. Lichti-Kaiser K, ZeRuth G, Jetten AM. Transcription factor GLI-similar 3 (GLIS3): implications fo the development of congenital hypothyroidism. JEndocrinol Diabetes Obes. 2014;2(2):1024.

29. Watanabe N, et al. A murine model of neonatal diabetes mellitus in Glis3-deficient mice. FEBS
Lett. 2009;583(12):2108-2113.

30. Kang HS, Beak JY, Kim YS, Herbert R, Jetten AM. Glis3 is associated with primary cilia and Wwtr1/ TAZ and implicated in polycystic kidney disease. Mol Cell Biol. 2009;29(10):2556-2569.

31. Kang HS, et al. Transcription Factor GLIS3: A New and Critical Regulator of Postnatal Stages of Mouse Spermatogenesis. Stem Cells. 2016;34(11):2772-2783.

32. Kang HS, et al. Transcription factor Glis3, a novel critical player in the regulation of pancreatic beta-cell development and insulin gene expression. Mol Cell Biol. 2009;29(24):6366-6379.

33. Kang HS, Takeda Y, Jeon K, Jetten AM. The spatiotemporal pattern of Glis3 expression indicates a regulatory function in bipotent and endocrine progenitors during early pancreatic development and in beta, PP and ductal cells. PLOS ONE. 2016;11(6):e0157138.

34. Yang Y, Chang BH, Chan L. Sustained expression of the transcription factor GLIS3 is required for normal beta cell function in adults. EMBO Mol Med. 2013;5(1):92-104.

35. Yang Y, et al. The Krüppel-like zinc finger protein GLIS3 transactivates neurogenin 3 for proper fetal pancreatic islet differentiation in mice. Diabetologia. 2011;54(10):2595-2605.

36. Boudellioua I, et al. Semantic prioritization of novel causative genomic variants. PLoS Comput Biol. 2017;13(4):e1005500.

37. Dimitri P. The role of GLIS3 in thyroid disease as part of a multisystem disorder. Best Pract Res Clin Endocrinol Metab. 2017;31(2):175-182.

38. van der Spek AH, Fliers E, Boelen A. The classic pathways of thyroid hormone metabolism [published online ahead of print January 18, 2017]. Mol Cell Endocrinol. https://doi.org/10.1016.jmce 2017.01.025.

39. Mansouri A, Chowdhury K, Gruss P. Follicular cells of the thyroid gland require Pax 8 gene function. Nat Genet. 1998;19(1):87-90.

40. Di Cosmo C, Liao XH, Dumitrescu AM, Philp NJ, Weiss RE, Refetoff S. Mice deficient in MCT8 reveal a mechanism regulating thyroid hormone secretion. J Clin Invest. 2010;120(9):3377-3388.

41. Saji M, Ringel MD. The PI3K-Akt-mTOR pathway in initiation and progression of thyroid tumors. Mol Cell Endocrinol. 2010;321(1):20-28.

42. Xu K, Liu P, Wei W. mTOR signaling in tumorigenesis. Biochim Biophys Acta. 2014;1846(2):638-654. 
43. Gérard AC, et al. Iodine deficiency induces a thyroid stimulating hormone-independent early phase of microvascular reshaping in the thyroid. Am J Pathol. 2008;172(3):748-760.

44. Kester MH, et al. Sulfation of thyroid hormone by estrogen sulfotransferase. JClin Endocrinol Metab. 1999;84(7):2577-2580.

45. Beak JY, Kang HS, Kim YS, Jetten AM. Functional analysis of the zinc finger and activation domains of Glis3 and mutant Glis3(NDH1). Nucleic Acids Res. 2008;36(5):1690-1702.

46. Ohno M, Zannini M, Levy O, Carrasco N, di Lauro R. The paired-domain transcription factor Pax 8 binds to the upstream enhancer of the rat sodium/iodide symporter gene and participates in both thyroid-specific and cyclicAMP-dependent transcription. Mol Cell Biol. 1999;19(3):2051-2060.

47. Riedel C, Levy O, Carrasco N. Posttranscriptional regulation of the sodium/ iodide symporter by thyrotropin. J Biol Chem. 2001;276(24):21458-21463.

48. Kogai T, Liu YY, Mody K, Shamsian DV, Brent GA. Regulation of sodium iodide symporter gene expression by Rac1/p38 $\beta$ mitogen-activated protein kinase signaling pathway in MCF-7 breast cancer cells. J Biol Chem. 2012;287(5):3292-3300.

49. Dentice $\mathrm{M}$, et al. Pendrin is a novel in vivo downstream target gene of the TTF-1/Nkx-2.1 homeodomain transcription factor in differentiated thyroid cells. Mol Cell Biol. 2005;25(22):10171-10182.

50. Huang K, Fingar DC. Growing knowledge of the mTOR signaling network. Semin Cell Dev Biol. 2014;36:79-90.

51. Pringle DR, et al. Follicular thyroid cancers demonstrate dual activation of PKA and MTOR as modeled by thyroid-specific deletion of Prkar1a and Pten in mice. JClin Endocrinol Metab. 2014;99(5):E804-E812.

52. Asaoka Y, Nagai Y, Namae M, Furutani-Seiki M, Nishina H. SLC7 family transporters control the establishment of left-right asymmetry during organogenesis in medaka by activating mTOR signaling. Biochem Biophys Res Commun. 2016;474(1):146-153.

53. Christie GR, Hajduch E, Hundal HS, Proud CG, Taylor PM. Intracellular sensing of amino acids in Xenopus laevis oocytes stimulates p70 S6 kinase in a target of rapamycin-dependent manner. J Biol Chem. 2002;277(12):9952-9957.
54. ZeRuth GT, Williams JG, Cole YC, Jetten AM. HECT E3 ubiquitin ligase itch functions as a novel negative regulator of Gli-Similar 3 (Glis3) transcriptional activity. PLOS ONE. 2015;10(7):e0131303.

55. ZeRuth GT, Yang XP, Jetten AM. Modulation of the transactivation function and stability of Krüppel-like zinc finger protein Gli-similar 3 (Glis3) by Suppressor of Fused. J Biol Chem. 2011;286(25):22077-22089.

56. Ferrara AM, et al. Changes in thyroid status during perinatal development of MCT8-deficient male mice. Endocrinology. 2013;154(7):2533-2541.

57. Levy O, et al. Characterization of the thyroid $\mathrm{Na}+$ /I- symporter with an anti-COOH terminus antibody. Proc Natl Acad Sci US A. 1997;94(11):5568-5573.

58. Takeda Y, Jothi R, Birault V, Jetten AM. ROR $\gamma$ directly regulates the circadian expression of clock genes and downstream targets in vivo. Nucleic Acids Res. 2012;40(17):8519-8535.

59. Narlikar L, Jothi R. ChIP-Seq data analysis: identification of protein-DNA binding sites with SISSRs peak-finder. Methods Mol Biol. 2012;802:305-322. 